\title{
A retrospective study on application of continuous renal replacement therapy for severe hand foot and mouth disease
}

\author{
Ha Manh Tuan ${ }^{1}$ and Do Chau Viet ${ }^{2}$ \\ ${ }^{1}$ University of Medicine and Pharmacy at HCM city, Vietnam \\ ${ }^{2}$ Children's Hospital 2 (33 Nguyen Du Street, District 1, HCM city), Vietnam
}

\begin{abstract}
Background: The patients with severe hand foot and mouth disease (HFMD) grade 4 will be indicated continuous renal replacement therapy (CRRT), but the mortality rate is still high. The study was conducted to show the application of CRRT for severe HFMD patients in earlier stage improving the mortality.
\end{abstract}

Method: A retrospective case series study was conducted about severe HFMD cases diagnosed clinically according to guidelines of the Ministry of Health and confirmed by Polymerase Chain Reaction (PCR) assay and having CRRT done. Variables would be recorded at admission, at starting CRRT and 16 hours later. The data were processed and analysed by software Stata version 14.0.

Results: There were 38 eligible cases included in the study. The mean age was 25.3 months; the male: female ratio was 1.5. Most cases admitted within the first 3 day of illness (94.7\%). The rate of HFMD grade 4 and grade 4 indicated CRRT was about $26.3 \%$ and $73.7 \%$ respectively. Vital signs, haematology parameters, biochemical parameters were improved significantly after 16 hours of CRRT. Average duration of CRRT was 2.4 \pm 0.7 days. The survival rate of cases with HFMD grade 3 was $82.1 \%$ higher than that of cases with HFMD grade 4 at $40 \%$ significantly. Overall survival rate of HFMD patients indicated CRRT was about $71 \%$.

Conclusion: The CRRT could be considered as one of the measures that contributed to the improvement of mortality in patients with severe HFMD, and if the CRRT is indicated early it can produce much better results.

\section{Introduction}

Hand foot and mouth disease (HFMD) is an infectious disease which is transmitted via faecal - oral route. This disease is caused by a group of enteroviruses of which Coxsackie A16 and Enterovirus A71 (EV A71) are more common [1,2]. Hand foot mouth disease occurs in many places around the world, but more popular in Western Pacific and Asian regions and can burst into large outbreaks [2]. In Viet Nam, HFMD is one of three most common types of infectious diseases with the prevalence ranging from 100.000 to 130.000 cases annually [3]. The disease is commonly diagnosed in children with the age of $1-3$ years and can lead to death if its severe complications are not managed appropriately $[1,2,4]$.

HFMD is classified into 4 grades based on its severity [3]. HFMD grade 1 consists of fever and skin lesions with or without oral lesions. HFMD grade 2 is HFMD with one of the following: myoclonic jerk, tremor, ataxia, limb weakness or lethargy. HFMD grade 3 is complicated HFMD with autonomous nervous system (ANS) dysregulation. HFMD grade 4 is HFMD grade 3 with any of the following: hypotension, pulmonary oedema, or heart failure. The patients with HFMD grade 1 , and grade 2 are uncomplicated HFMD which are supportively managed and closely monitored if the patient presents with signs of central nervous system involvement. The patients with HFMD grade 3 are monitored in intensive care unit and indicated with intravenous immunoglobulin (IVIG), dobutamine, and milrinone.
The HFMD patients grade 4 are supported by ventilator, vasoactive drugs, administered with IVIG and indicated with continuous renal replacement therapy (CRRT) [3].

So far, there have been few studies reporting the application of CRRT for patients with HFMD grade $4[5,6]$. The preliminary results from application of CRRT for patients with HFMD grade 4 were encouraging although the mortality rate was still high [5]. This study was conducted to show the effectiveness of CRRT in earlier stage of severe HFMD patients.

\section{Methods and materials}

\section{Study design}

We conducted a retrospective study to collect data from severe EVA71 HFMD patients with cardiopulmonary complications, admitted to the Infectious Diseases Ward, Children's Hospital 2 from 2011-2015

${ }^{\star}$ Correspondence to: Ha Manh Tuan, University of Medicine and Pharmacy at HCM city, Vietnam, Tel: 0903311709, E-mail: hamanhtuan@ump.edu.vn

Key words: hand foot mouth disease, continuous renal replacement therapy

Received: December 06, 2019; Accepted: December 27, 2019; Published: December 30, 2019 
and received CRRT. Patients with chronic diseases or missing data were excluded from the database. The study was approved by the Institutional Review Board (IRB) of Children's Hospital 2. The informed consent form was waived, as we use unidentified retrospective data.

\section{Patients}

Patients were diagnosed as grade 3 and 4 according to the Vietnam $\mathrm{MoH}$ guidelines (as described above). All cases were EV-A71 positive by real time RT-PCR and received CRRT plus conventional treatment, as mentioned in the guidelines.

The conventional treatments include immunoglobulin $1 \mathrm{~g} / \mathrm{kg} / \mathrm{dose}$ (the second dose could be considered based on patient's condition); phenobarbital $10-20 \mu \mathrm{g} / \mathrm{kg}$ in 30 minutes, milrione $0.4 \mu \mathrm{g} / \mathrm{kg} / \mathrm{min}$ in up to 72 hours; dobutamine $5 \mu \mathrm{g} / \mathrm{kg} / \mathrm{min}$ to maximum $20 \mu \mathrm{g} / \mathrm{kg} /$ min; mechanical ventilator and normal saline or lactate ringer $5 \mathrm{ml} /$ $\mathrm{kg} / 15$ mins in patients with shock. If patients were suspected to have nosocomial infection or sepsis, antibiotics were applied.

Patients with HFMD grade 3 were treated with above conventional therapy and monitored every 1 hour in the intensive care unit. If the patients did not improve after 24 hours of conventional treatment, CRRT would be indicated. Patients with HFMD grade 4 on admission or becoming worse into HFMD grade 4 during close monitoring in the intensive care unit would be indicated CRRT [3].

The CRRT was carried out with Prima Flex machine, hemofilter M60 - M100, substitution fluid Hemosol. Vascular access was done with double lumen $6.5-8 \mathrm{~F}$ catheter. Continuous venous - venous hemofiltration $(\mathrm{CVVH})$ mode was chosen for the patients. The rate of infusion pump was averaged $5 \mathrm{ml} / \mathrm{kg} / \mathrm{min}$. The ratio of the pre- to post- dilution was 1:2. The filter circuit was pretreated with saline that contained unfractionated heparin and during $\mathrm{CVVH}$, heparin was used with an infusion of average $15 \mathrm{UI} / \mathrm{kg} / \mathrm{h}$. The activated partial thromboplastin time (APTT) was tested every 8 hours.

\section{Data collection and analysis}

Data from eligible cases was obtained from hospital records and entered to the electronic database, using standard case report form (CRF). Key variables were defined prior to data collection and included demographic data, clinical signs, vital signs, severity of disease, indication of CRRT, laboratory parameters such as biochemistry, liver and kidney function, cardiac enzymes, electrolytes, coagulation, glycemia and patient's outcome.

The variables were recorded at admission, the beginning of CRRT, and every 8 hours after CRRT application.

Data were analysed by Stata software version 14.0. Continuous and category variables were described by mean and percentage, respectively. Chi square test was applied to compare two percentages, and t test for 2 means.

\section{Results}

\section{Demographic characteristics of patients}

There were 38 eligible patients enrolled in the study within 4 years from $6 / 2011-12 / 2015$. The mean age was 25.3 months with $86.6 \%$ of cases reported in children younger than 3 years old. The male: female ratio was 1.5. Most of patients lived in Ho Chi Minh (HCM) city and neighbouring provinces (73.6\%). There were $100 \%$ of cases isolated Enterovirus A71 with PCR technique from at least one of either throat swabs or rectal swabs, $87 \%$ of rectal swabs and $92 \%$ of throat swabs yielded the result of Enterovirus A71 (Table 1).

\section{Indications of continuous renal replacement therapy}

The rate of cases with HFMD grade 4 indicated CRRT was only $26.3 \%$, this is around three times less than the rate of cases with HFMD grade 3 having CRRT given. Indications of CRRT for the HFMD patients grade 3 included diminished consciousness, ANS dysregulation, and abnormal breathing (table 2).

\section{Effects of continuous renal replacement therapy}

The vital signs of severe HFMD patients improved significantly 16 hours after the initiation of CRRT (Table 3 ). The patient's temperature was about $38.8^{\circ} \mathrm{C}$ at admission, rose over $39^{\circ} \mathrm{C}$ when the patients' conditions turned worse and remained high until starting CRRT. The patients' temperature declined about $1^{\circ} \mathrm{C} 4$ hours after initiation of CRRT, dropped gradually and became stable since the twelfth hour of CRRT (Figure 1). The patients' mean pulse reached a peak of 180 $\mathrm{ppm}$ at the beginning of CRRT, it decreased by $10 \mathrm{ppm}$ relevant to the drop of $1^{\circ} \mathrm{C}$ in the mean temperature at the fourth hour of CRRT, then maintained $150 \mathrm{ppm}$ since the sixteenth hour (Figure 2). In term of mean systolic arterial pressure, it declined $10 \mathrm{mmHg} 2$ hours after the CRRT initiated, returned to normal at the eighth hour of CRRT, and became stable at the sixteenth hour (Figure 3).

Similarly, the laboratory results had better changes 16 hours after the initiation of CRRT. The serum glucose, troponin, creatinine, CRP, ALT level improved significantly at the sixteenth hour of CRRT (Table 3).

\section{Outcome of continuous renal replacement therapy}

The mean duration of CRRT was $2.4 \pm 0.7$ days, it was $2.8 \pm 0.4$ days for survivor. The rate of survivor in HFMD patients grade 3 was double higher than that in HFMD patients grade 4 ( $\mathrm{p}=0.015, \chi^{2}$ test) (Table 4$)$.

\section{Discussion}

Thirty-eight cases of severe HFMD were enrolled, all of them were isolated Enterovirus 71 from either throat swab or rectal swab with

Table 1. Demographic characteristic of the patients

\begin{tabular}{|c|c|c|}
\hline & Number of cases & Rate (\%) \\
\hline \multicolumn{3}{|l|}{ Gender } \\
\hline Male & 23 & 60.5 \\
\hline Female & 15 & 39.5 \\
\hline Age (months) & \multicolumn{2}{|c|}{$25.3 \pm 8.2$} \\
\hline \multicolumn{3}{|l|}{ Residency } \\
\hline Ho Chi Minh city & 11 & 28.9 \\
\hline Binh dương & 11 & 28.9 \\
\hline Dong Nai & 6 & 15.8 \\
\hline Others & 10 & 26.4 \\
\hline \multicolumn{3}{|l|}{ Sources of transmission } \\
\hline Kindergarten & 16 & 42.1 \\
\hline Home & 22 & 57.9 \\
\hline Length of illness $<3$ days before admission & 36 & 94.7 \\
\hline Enterovirus A71 isolates & 38 & 100 \\
\hline
\end{tabular}

Table 2. Indication of continuous renal replacement therapy

\begin{tabular}{|l|c|c|}
\hline & Number of cases & $\begin{array}{c}\text { Rate } \\
\text { (\%) }\end{array}$ \\
\hline Grade 4 & 10 & 26.3 \\
\hline Grade 3 & 28 & 73.7 \\
\hline Diminished consciousness & 10 & 26.3 \\
\hline ANS dysregulation & 11 & 28.9 \\
\hline Abnormal breathing & 7 & 18.4 \\
\hline
\end{tabular}

ANS: Autonomous Nervous System 


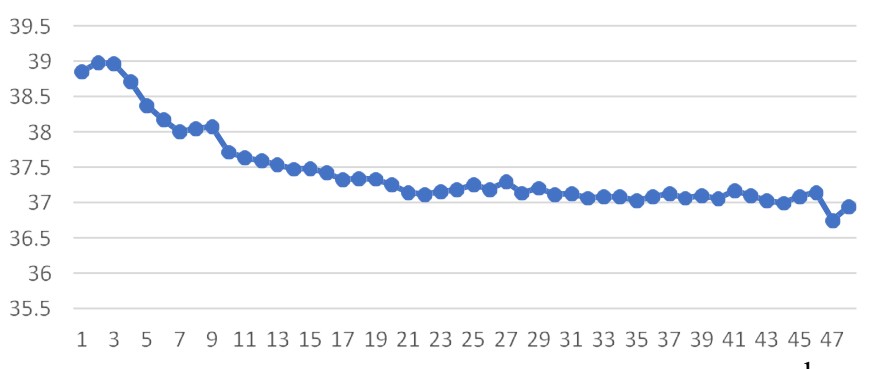

Figure 1. Change in the patients' mean temperature in time of CRRT

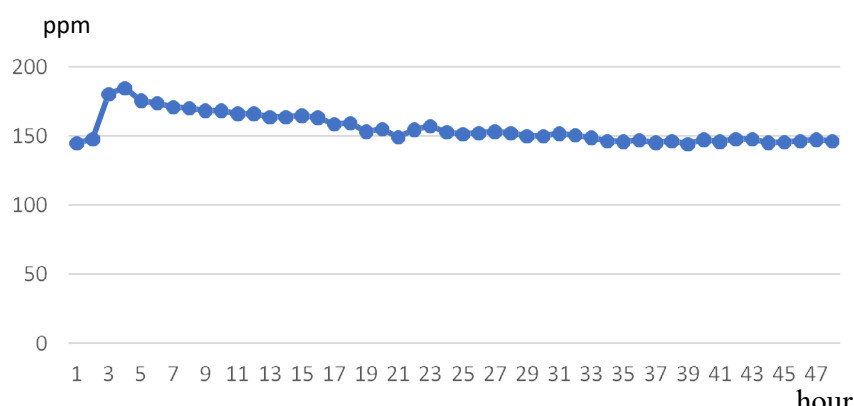

Figure 2. Change in the patients' mean pulse in time of CRRT

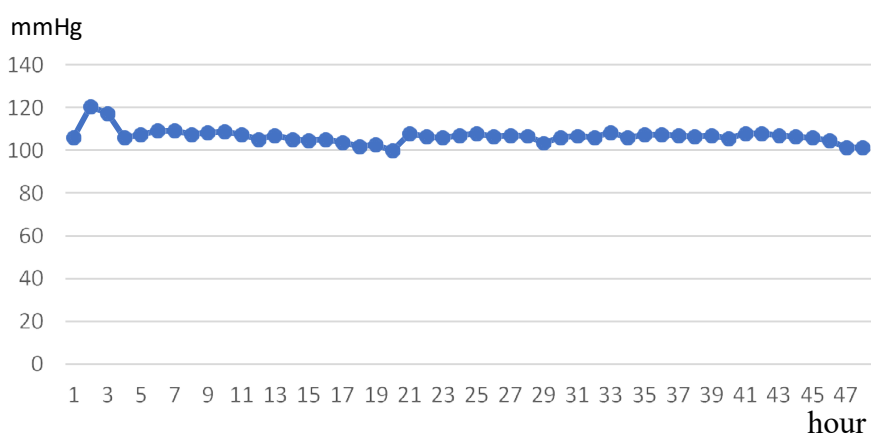

Figure 3. Change in the patients' mean systolic arterial pressure in time of CRRT

Table 3. Changes in vital signs and laboratory results between starting continuous renal replacement therapy and 16 hours later

\begin{tabular}{|l|c|c|c|}
\hline & Starting CRRT & 16 hours later & P $(\mathrm{t}$-test $)$ \\
\hline Vital signs & $180.3 \pm 27.5$ & $159.2 \pm 26.3$ & $<0.05$ \\
\hline Pulse $(\mathrm{ppm})$ & $39.0 \pm 1.1$ & $37.5 \pm 1.0$ & $<0.05$ \\
\hline Temperature $\left({ }^{\circ} \mathrm{C}\right)$ & $120.0 \pm 28.0$ & $109.2 \pm 24.1$ & $<0.05$ \\
\hline $\begin{array}{l}\text { Systolic blood pressure } \\
(\mathrm{mmHg})\end{array}$ & & & \\
\hline Laboratory parameters & $16.1 \pm 6.8$ & $12.1 \pm 4.2$ & $<0.05$ \\
\hline WBC $\left(\mathrm{x} 1000 / \mathrm{mm}^{3}\right)$ & $382.6 \pm 114.0$ & $189.7 \pm 60.9$ & $<0.05$ \\
\hline Platelet count $\left(\mathrm{x} 1000 / \mathrm{mm}^{3}\right)$ & $11.0 \pm 1.6$ & $9.6 \pm 1.7$ & $>0.05$ \\
\hline Hemoglobin $(\mathrm{g} / \mathrm{l})$ & $150.0 \pm 88.5$ & $133.6 \pm 74.0$ & $<0.05$ \\
\hline Glycemia $(\mathrm{mg} / \mathrm{l})$ & $3.9 \pm 3.9$ & $2.7 \pm 2.8$ & $<0.05$ \\
\hline Troponin $(\mathrm{ng} / \mathrm{ml})$ & $2,2 \pm 1,3$ & $2.0 \pm 0.7$ & $>0.05$ \\
\hline Lactate $(\mathrm{mmol} / \mathrm{l})$ & $5,6 \pm 2,4$ & $4.1 \pm 1.3$ & $<0.05$ \\
\hline Creatinine $(\mathrm{mg} / \mathrm{l})$ & $15.2 \pm 23.0$ & $8.5 \pm 5.9$ & $<0.05$ \\
\hline CRP $(\mathrm{mg} / \mathrm{l})$ & $66.2 \pm 44.1$ & $62.7 \pm 42.0$ & $>0.05$ \\
\hline AST $(\mathrm{U} / \mathrm{l})$ & $27.0 \pm 16.9$ & $17.0 \pm 3.6$ & $<0.05$ \\
\hline ALT $(\mathrm{U} / \mathrm{l})$ & $\mathrm{As}$ & \\
\hline
\end{tabular}

CRP: C-reactive protein; AST: Aspartate amino transferase; ALT: Alanine amino transferase; WBC: White Blood Cell.
Table 4. Outcome of continuous renal replacement therapy for severe HFMD patients

\begin{tabular}{|c|c|c|c|}
\hline & Number of cases & Survivor & Rate of survivor (\%) \\
\hline Grade 3 & 28 & 23 & 82.1 \\
\hline Grade 4 & 10 & 4 & 40.0 \\
\hline Total & 38 & 27 & 71.0 \\
\hline
\end{tabular}

PCR technique. Most of them were under 3 years old, the morbidity was higher in male than in female, and most of them were admitted within the first 3 day of illness. These shown that the severe HFMD cases are almost caused by Enterovirus 71, mainly occur in young children and progress rapidly within the first few days of illness. These have also been reported in many previous studies $[1,4,5]$.

According to a guideline of Ministry of Health developed in 2011 [3], the HFMD cases are classified as grade 4 when the patients present with one of the following conditions: shock, pulmonary oedema, central cyanosis or grasping breathing, whereas the HFMD cases grade 3 include signs of ANS dysregulation such as tachycardia 150 $-170 \mathrm{ppm}$, hypertension, profuse sweating, respiratory abnormalities (tachypnoea, laboured breathing). The CRRT is only indicated for the severe HFMD patients grade 4, but the HFMD cases grade 3 have not yet been recommended the CRRT. However, we have realized that if the CRRT is initiated for HFMD patients grade 4 , the mortality rate will be very high, whereas the outcome will be better if the CRRT is early indicated for the HFMP patients grade 3 who have presented with the signs of ANS dysregulation. From our clinical observation, we apply the CRRT to HFMD cases grade 3 who do not respond well to relevant treatments following the guideline [4]. So, the rate of CRRT for severe HFMD cases grade 3 was much higher than that for severe HFMD cases grade 4 in this study. The overall survival rate of severe HFMD cases indicated CRRT is much better in our study than in NMTien's study [5]. In this study, the author indicated CRRT mainly for severe HFMD patients grade $4(90 \%)$ and the mortality rate was approximately double higher in this study than in our study.

The study shown the manifestation of ANS dysregulation such as high fever, tachypnoea, arterial hypertension improved remarkably 16 hours after starting CRRT. Similarly, the laboratory abnormalities got better after 16 hours of CRRT. All cases with pulmonary oedema have ameliorated at the same time. The mechanism of CRRT resulting a good outcome in the severe HFMD patients remained unclear. Although, the effect of CRRT for patients with multiple organ failure caused by sepsis has been verified through the capacity of CRRT to remove mediators. The recent studies have shown that the HFMD patients with fulminant clinical manifestations have probably been associated with the production of inflammatory cytokine and chemokines caused by EV 71 infection [7-10]. The fact that the CRRT can withdraw inflammatory mediators from the blood by a convective mechanism and/or by adsorption to the membrane of the hemofilter may be an explanation for the effectiveness of CRRT in the management of severe HFMD patients $[11,12]$.

Besides the appropriate indication of CRRT for the severe HFMD patients, the other factors have might contributed to the effectiveness of CRRT in management of the severe HFMD cases. They are that the medical equipment is always available, the skilled staff are ready to carry out the CRRT at any time, the preparation for performing CRRT must be so rapid to get valuable time to save the patients who deteriorate rapidly, and at last the staff's competency deals with complications of severe HFMD and problems occurring during implementing CRRT. 


\section{Conclusion}

This study has shown the effectiveness of CRRT in improving clinical signs and laboratory measurements in the HFMD patients with fulminant complications. The CRRT may be considered as a measure contributing to make the outcome of the severe HFMD patients better. The result is much better if the CRRT is indicated early in the severe HFMD patients grade 3 who do not respond well to the appropriate treatments. However, the early indication of CRRT for the severe HFMD patients needs further studies.

\section{References}

1. Li-Mei S, Huan-Ying Z, Hui-Zhen Z, He JF, et al. (2008) An Enterovirus 71 epidemic in Guandong Province of China, 2008: Epidemiologic, Clinical and Virologic manifestations". Jpn J Infect Dis 64: 13-18. [Crossref]

2. World Health Organization. A guide to clinical management and public health response for hand, foot and mouth (HFMD). 2011; URL: http://www.wpro.who.int/publications/ docs/ Guidance for the clinical management of HFMD.pdf [Accessed 20 December 2017].

3. Ministry of Health, Viet Nam (2011) A Guideline for Diagnosis and Treatment of Hand Foot Mouth Disease". https://thuvienphapluat.vn/van-ban/The-thao-Y-te/Quyet-dinh2554-QD-BYT-huong-dan-chan-doan-dieu-tri-benh-tay-chan-mien-126821.aspx. [Accessed 20 December 2017].

4. Kim KH (2010) Enterovirus 71 infection: An experience in Korea, 2009. Korean J Pediatr 53: 616-622. [Crossref]
5. Tien NM, Quang PV, Ut TH (2014) Factors related to the outcome of continuous renal replacement therapy for hand foot mouth disease with severe complications. Journal of Medicine HCM city 12: 98 -106.

6. Phan PH, Dau HV, Chu ST, Phung TB, Van Pham T, et al. (2012) Continuous veno-venous hemofiltration for treatment of enterovirus 71-induced fulminant cardiopulmonary failure: a case report. J Med Case Rep 6: 159. [Crossref]

7. Lin TY, Hsia SH, Huang YC, Wu CT, Chang LY (2003) Proinflammatory cytokine reactions in Enterovirus 71 infections of the central nervous system. Clin Infect Dis 36: 269-274. [Crossref]

8. Wang SM, Lei HY, Liu CC (2012) Cytokine immunopathogenesis of Enterovirus 71 Brain Stem Encephalitis. Clin Dev Immunol 12: 876241. [Crossref]

9. Wang SM, Lei HY, Huang KJ, Wu JM, Wang JR, et al. (2003) Pathogenesis of enterovirus 71 brainstem encephalitis in pediatric patients: roles of cytokines and cellular immune activation in patients with pulmonary edema. $J$ Infect Dis 188: 564570. [Crossref]

10. Wang SM, Lei HY, Yu CK, Wang JR, Su IJ, et al. (2008) Acute chemokine response in the blood and cerebrospinal fluid of children with enterovirus 71-associated brainstem encephalitis. J Infect Dis 198: 1002-1006. [Crossref]

11. Goldstein SL, Somers MJ, Baum MA, Symons JM, Brophy PD, et al. (2007) Pediatric patients with multi-organ dysfunction syndrome receiving continuous renal replacement therapy. Kidney Int 67: 653-658. [Crossref]

12. Peng Z, Pai P, Hong-Bao L, Rong L, Han-Min W, et al. (2010) The impacts of continuous veno-venous hemofiltration on plasma cytokines and monocyte human leukocyte antigen DR expression in septic patients. Cytokine 50: 186-191. [Crossref]

Copyright: (2019 Tuan HM. This is an open-access article distributed under the terms of the Creative Commons Attribution License, which permits unrestricted use, distribution, and reproduction in any medium, provided the original author and source are credited. 